\title{
Gravitating gauged BPS baby Skyrmions
}

\author{
M. Wachla \\ Institute of Nuclear Physics, PAN ul. Radzikowskiego 152, 31-342 Kraków, Poland
}

(Received 17 April 2018; published 15 March 2019)

\begin{abstract}
We show that the minimally gauged Bogomol'nyi-Prasad-Sommerfield baby Skyrme model remains a Bogomol'nyi-Prasad-Sommerfield theory after coupling with the gravity. That is, the topologically nontrivial configurations called the baby Skyrmions and carrying a magnetic flux are solutions to a zero pressure equation. It follows that the proper mass, the magnetic flux, and the proper geometric volume are the linear functions of the topological charge, while the total Arnowitt-Deser-Misner mass and geometric radius receive a contribution due to the gravitational interaction that is quadratic in the topological charge. All these quantities are found exactly as target space integrals (averages) of the so-called superpotential. A complete classification of the possible mass-radius curves is provided. As an example, we consider the model with the pionlike mass potential, for which an approximated but analytical form of the superpotential is provided.
\end{abstract}

DOI: $10.1103 /$ PhysRevD.99.065006

\section{INTRODUCTION}

Magnetized gravitating matter in the $(2+1)$ dimensions could be viewed as a toy model of magnetic compact stars in the $(3+1)$-dimensional spacetime. If this matter can be related to a low energy regime of $\mathrm{QCD}$, for example, by means of an effective action, then we arrive at a model of magnetized neutron stars.

This goal can be realized within the framework of the $(3+1)$-dimensional Skyrme model [1], which is one of the most acceptable effective models of baryons, atomic nuclei, and nuclear matter. In fact, in its so-called Bogomol'nyiPrasad-Sommerfield (BPS) limit [2] (also [3]), the Skyrme model was shown to be able to support the neutron stars with the observables (maximal mass, maximal radius, mass-radius curve, etc.) in a very good agreement with (still poor) observational data [4]. Such gravitating solitonic solutions go much further than a usual charge one gravitating Skyrmion [5], as the topological charge of the maximal mass solution is of the order $10^{57}$. This result was achieved due to the BPS nature of the model and a large moduli space of static solutions forming the group of volume preserving diffeomorphisms-both closely related to the most crucial features of nuclear matter: its very small binding energies and the liquidlike nature. Although in such a BPS limit the usual part of the Skyrme model is neglected, the obtained results should

Published by the American Physical Society under the terms of the Creative Commons Attribution 4.0 International license. Further distribution of this work must maintain attribution to the author(s) and the published article's title, journal citation, and DOI. Funded by SCOAP ${ }^{3}$. give good approximations to the bulk quantities, as the BPS part of the full model provides the leading contribution at high density and pressure, which is the case inside neutron stars [6]. Of course, at some point the non-BPS part of the Skyrme model should also be taken into account. This is a difficult task, because Skyrmions in the full model possess very complicated geometric shapes with only the discrete symmetries [7], rendering the problem of the computation of self-gravitating multi-Skyrmions too complicated. Then, only a mean field approach seems to be applicable [8]. This concerns the vector meson Skyrme model [9] as well as the weakly bound Skyrme model [10].

In the next step, one should couple the (BPS) Skyrme model with the Maxwell field, which requires the introduction of the usual covariant derivative and the inclusion of the Maxwell as well as the Wess-Zumino-Witten term [11]. Already the Maxwell contribution (no gravity) breaks all nice properties of the BPS model, rendering the analytical computation impossible.

However, in the $(2+1)$ dimensions the situation is much better. First of all, there is a lower dimensional counterpart of the Skyrme model, known as the baby Skyrme model [12], which also possesses the BPS sectors. Especially, the BPS baby Skyrme model [13-16] is a lower dimensional version of the BPS Skyrme model. This BPS theory can be minimally gauged without destroying its BPS nature $[17,18]$. That is to say, the gauged (strictly speaking magnetic) solitons (baby Skyrmions) are solutions of certain Bogomol'nyi equations and, therefore, saturate a pertinent topological bound. Furthermore, the matter is still of a perfect fluid type. Second, it has been shown very recently that its coupling to the gravity also preserves the BPS property of the BPS baby Skyrme model [19]. This allowed for an analytical 
computation of gravitating baby Skyrmions in the asymptotically flat spacetime. Therefore, the natural question arises, what happens if both interactions (Maxwell and gravity) are added to the BPS baby Skyrme model simultaneously? If the gravitating gauged BPS baby Skyrme model remains a BPS theory, we get a unique opportunity to study the magnetic planar solitons (a toy model of magnetars) in an analytical way.

It is the main aim of the present work to study the BPS property of the gauged BPS baby Skyrme model after coupling it to the gravity. In particular, we check how the maximal Arnowitt-Deser-Misner (ADM) mass as well as the ADM mass-radius relation for the gravitating BPS planar Skyrmions are modified if the magnetic field is included.

To accomplish this program in an analytical manner, we find a way to solve the so-called superpotential equation, i.e., a nonlinear differential equation defining a target space function (superpotential) that enters expressions for all observables. Our method allows us to construct this function with an arbitrary precision.

\section{THE GRAVITATING GAUGED BPS BABY SKYRME MODEL}

\section{A. Static and axially symmetric field equations}

The gravitating gauged BPS baby Skyrme model is given by the following action where the metric tensor is treated as a dynamical quantity:

$$
\begin{aligned}
S= & S_{04}+S_{\mathrm{EH}} \\
= & \int d^{3} x|g|^{\frac{1}{2}}\left(-\lambda^{2} \pi^{2}|g|^{-1} g_{\alpha \beta} \tilde{\mathcal{B}}^{\alpha} \tilde{\mathcal{B}}^{\beta}-\mu^{2} \mathcal{U}-\frac{1}{4 e^{2}} F_{\mu \nu}^{2}\right) \\
& +S_{\mathrm{EH}},
\end{aligned}
$$

where $S_{\mathrm{EH}}$ is the Einstein-Hilbert (EH) action in the $(2+1)$-dimensional spacetime. Here

$$
\tilde{\mathcal{B}}^{\mu}=\frac{1}{8 \pi} \epsilon^{\mu \nu \rho} \vec{\phi} \cdot\left(D_{\nu} \vec{\phi} \times D_{\nu} \vec{\phi}\right)
$$

is a gauge invariant version of the topological current

$$
\mathcal{B}^{\mu}=\frac{1}{8 \pi} \epsilon^{\mu \nu \rho} \vec{\phi} \cdot\left(\vec{\phi}_{\nu} \times \vec{\phi}_{\rho}\right) .
$$

The $U(1)$ gauging is performed in the usual way, i.e., by promoting the global $U(1)$ symmetry of the BPS baby Skyrme model to a local one. This means that we change the usual derivatives to their covariant versions [20] (for the general baby Skyrmions with magnetic field see [21,22])

$$
D_{\mu} \vec{\phi}=\vec{\phi}_{\mu}+A_{\mu} \vec{n} \times \vec{\phi}
$$

Furthermore, $\vec{\phi}$, i.e., the baby Skyrme field, is a unit three component isovector $\vec{\phi} \in \mathbb{S}^{2}$ ( $\mathbb{S}^{2}$ denotes a two-sphere) and the constant vector $\vec{n}=(0,0,1)$. The potential $\mathcal{U}$ is assumed to be a one-vacuum potential that depends only on the third component of the matter field; i.e., it has a single zero at $\vec{\phi}=\vec{\phi}_{0}$ where $\vec{\phi}_{0}=\vec{n} . F_{\mu \nu}$ is the usual field strength tensor of the $U(1)$ Maxwell field $A_{\mu}$ in the $(2+1)$ dimensions.

The corresponding Einstein equations are

$$
G_{\alpha \beta}=\frac{\kappa^{2}}{2} T_{\alpha \beta},
$$

where $\kappa^{2}=16 \pi G$ and $G$ is the three-dimensional gravity constant. In the subsequent analysis, we assume the axial symmetry for the metric

$$
d s^{2}=\mathbf{A}(r) d t^{2}-\mathbf{B}(r) d r^{2}-r^{2} d \varphi^{2},
$$

which gives the following Einstein tensor $G_{\mu \nu}\left(\mathbf{A}_{r}=\partial_{r} \mathbf{A}\right.$, etc.):

$$
\begin{aligned}
G_{00} & =\frac{1}{2 r} \frac{\mathbf{A} \mathbf{B}_{r}}{\mathbf{B}^{2}}, \quad G_{11}=\frac{1}{2 r} \frac{\mathbf{A}_{r}}{\mathbf{A}}, \\
G_{22} & =-\frac{r^{2}}{4}\left(\frac{\mathbf{A}_{r}}{\mathbf{A}} \frac{\mathbf{B}_{r}}{\mathbf{B}^{2}}+\frac{1}{\mathbf{B}}\left(\frac{\mathbf{A}_{r}^{2}}{\mathbf{A}^{2}}-\frac{2 \mathbf{A}_{r r}}{\mathbf{A}}\right)\right),
\end{aligned}
$$

and the curvature scalar $\mathbf{R}$,

$\mathbf{R}=\frac{-r \mathbf{B} \mathbf{A}_{r}^{2}-2 \mathbf{A}^{2} \mathbf{B}_{r}+A\left(-r \mathbf{A}_{r} \mathbf{B}_{r}+2 \mathbf{B}\left(\mathbf{A}_{r}+r \mathbf{A}_{r r}\right)\right)}{2 r \mathbf{A}^{2} \mathbf{B}^{2}}$.

This assumption comes from the observation that the gauged BPS baby Skyrme model has the ground state solutions (in each topological sector) in such an axially symmetric form. In fact, these energy minimizers enjoy a huge degeneracy, which is the group of the area preserving diffeomorphisms.

Next we observe that the energy-momentum tensor has two contributions,

$$
T^{\alpha \beta}=T_{m}^{\alpha \beta}+T_{e m}^{\alpha \beta},
$$

where we have the matter part

$T_{m}^{\alpha \beta}=2 \lambda^{2} \pi^{2}|g|^{-1} \tilde{\mathcal{B}}^{\alpha} \tilde{\mathcal{B}}^{\beta}-\left(\lambda^{2} \pi^{4}|g|^{-1} g_{\mu \nu} \tilde{\mathcal{B}}^{\mu} \tilde{\mathcal{B}}^{\nu}-\mu^{2} \mathcal{U}\right) g^{\alpha \beta}$

and the electromagnetic part

$$
T_{e m}^{\alpha \beta}=\frac{1}{e^{2}}\left(\frac{1}{4} g^{\alpha \beta} F_{\mu \nu} F^{\mu \nu}-F^{\alpha \nu} F^{\beta}{ }_{\nu}\right) .
$$

Let us begin with the baby Skyrme contribution. After coupling to gravity the energy-momentum tensor still possesses the perfect fluid form 


$$
T_{m}^{\alpha \beta}=(p+\rho) u^{\alpha} u^{\beta}-p g^{\alpha \beta},
$$

where the proper energy density and pressure are

$$
\begin{aligned}
& \rho=\lambda^{2} \pi^{2}|g|^{-1} g_{\mu \nu} \tilde{\mathcal{B}}^{\mu} \tilde{\mathcal{B}}^{\nu}+\mu^{2} \mathcal{U}, \\
& p=\lambda^{2} \pi^{2}|g|^{-1} g_{\mu \nu} \tilde{\mathcal{B}}^{\mu} \tilde{\mathcal{B}}^{\nu}-\mu^{2} \mathcal{U},
\end{aligned}
$$

while the four velocity is

$$
u^{\alpha}=\frac{\tilde{\mathcal{B}}^{\alpha}}{\sqrt{g_{\mu \nu} \tilde{\mathcal{B}}^{\mu} \tilde{\mathcal{B}}^{\nu}}} .
$$

For the static configurations it simplifies to

$$
T^{00}=\rho g^{00}, \quad T^{i j}=-p g^{i j},
$$

where we also assumed that there is no electric field

$$
A_{\mu}=\left(0, A_{1}(\vec{x}), A_{2}(\vec{x})\right) .
$$

Now, consistently with the assumptions on the metric we will restrict ourselves to an axially symmetric matter and gauge field. This means that

$$
A_{0}=A_{r}=0, \quad A_{\phi}=n a(r),
$$

while for the baby Skyrme field expressed by the stereographic projection

$$
\vec{\phi}=\frac{1}{1+|u|^{2}}\left(u+\bar{u},-i(u-\bar{u}), 1-|u|^{2}\right)
$$

we apply the following ansatz:

$$
u=f(r) e^{i n \varphi}, \quad h=1-\frac{1}{1+f^{2}} .
$$

All this leads to the following expressions for the baby Skyrme energy density and pressure:

$$
\begin{aligned}
& \rho=\frac{\lambda^{2} n^{2}}{4 \mathbf{B} r^{2}}(1+a)^{2} h_{r}^{2}+\mu^{2} \mathcal{U}, \\
& p=\frac{\lambda^{2} n^{2}}{4 \mathbf{B} r^{2}}(1+a)^{2} h_{r}^{2}-\mu^{2} \mathcal{U},
\end{aligned}
$$

while

$$
\tilde{B}^{0}=-\frac{n}{2 \pi}(1+a) h_{r}
$$

Note that our topological current differs by a factor of $1 / r \sqrt{\mathbf{B}}$ from the usual topological charge density $q$. This is a consequence of our convention to extract the metric factor from the antisymmetric tensor. Hence $n=\int \operatorname{vol}_{\mathbb{R}^{2}} \frac{1}{r \sqrt{\mathbf{B}}} B^{0}=-\int r \sqrt{\mathbf{B}} d r d \varphi \frac{n}{2 \pi r \sqrt{\mathbf{B}}} h_{r}$

while

$$
n=\int d r d \varphi B^{0} .
$$

In addition, the electromagnetic part of the energy-stress tensor reads (diagonal terms)

$$
\begin{aligned}
T_{e m}^{00} & =\frac{n^{2}}{2 e^{2}} \frac{1}{\mathbf{A} \mathbf{B} r^{2}} a_{r}^{2}, \\
T_{e m}^{r r} & =\frac{n^{2}}{2 e^{2}} \frac{1}{\mathbf{B}^{2} r^{2}} a_{r}^{2}, \\
T_{e m}^{\varphi \varphi} & =\frac{n^{2}}{2 e^{2}} \frac{1}{\mathbf{B} r^{4}} a_{r}^{2} .
\end{aligned}
$$

Now, we can write the Einstein equations in a compact form,

$$
\begin{aligned}
\frac{\mathbf{B}_{r}}{\mathbf{B}} & =\kappa^{2} r \mathbf{B} \tilde{\rho}, \\
\frac{\mathbf{A}_{r}}{\mathbf{A}} & =\kappa^{2} r \mathbf{B} \tilde{p}, \\
(\tilde{p} \mathbf{B})_{r} & =\kappa^{2} r \mu^{2} \mathbf{B}^{2} \mathcal{U} \tilde{p},
\end{aligned}
$$

where the matter density and pressure, with the gauge component included, read

$$
\begin{aligned}
& \tilde{\rho}=\frac{n^{2}}{2 e^{2} r^{2} \mathbf{B}} a_{r}^{2}+\frac{\lambda^{2} n^{2}}{4 r^{2} \mathbf{B}}(1+a)^{2} h_{r}^{2}+\mu^{2} \mathcal{U}, \\
& \tilde{p}=\frac{n^{2}}{2 e^{2} r^{2} \mathbf{B}} a_{r}^{2}+\frac{\lambda^{2} n^{2}}{4 r^{2} \mathbf{B}}(1+a)^{2} h_{r}^{2}-\mu^{2} \mathcal{U} .
\end{aligned}
$$

This set of equations has to be supplemented by the pertinent Maxwell equations

$$
\frac{1}{e^{2}} \partial_{\nu}\left(\sqrt{g} g^{\mu \alpha} F_{\alpha \beta} g^{\beta \nu}\right)=J^{\mu},
$$

where $J^{\mu}$ is the current due to the covariant derivative in the matter part of the model. It reads

$$
J^{\mu}=\lambda^{2} \pi^{2}|g|^{-1 / 2} g_{\alpha \beta} \frac{\partial}{\partial A_{\mu}} \tilde{B}^{\alpha} \tilde{B}^{\beta} .
$$

Hence,

$$
\frac{n}{e^{2}} \partial_{r}\left(\sqrt{\frac{\mathbf{A}}{\mathbf{B}}} \frac{a_{r}}{r}\right)=J^{\phi},
$$


where

$$
J^{\phi}=\lambda^{2} \pi^{2}|g|^{-1 / 2} g_{00} \frac{\partial}{\partial A_{\phi}} \tilde{B}^{0} \tilde{B}^{0}=\lambda^{2} \sqrt{\frac{\mathbf{A}}{\mathbf{B}}} \frac{n}{2}(1+a) \frac{h_{r}^{2}}{r} .
$$

Together we get

$$
\frac{n}{e^{2}} \partial_{r}\left(\sqrt{\frac{\mathbf{A}}{\mathbf{B}}} \frac{a_{r}}{r}\right)=\lambda^{2} \sqrt{\frac{\mathbf{A}}{\mathbf{B}}} \frac{n}{2}(1+a) \frac{h_{r}^{2}}{r} .
$$

So, finally we are left with a system of the four ordinary differential equations (2.28), (2.29), (2.30), and (2.37) for four unknown functions: the two metric functions $\mathbf{B}, \mathbf{A}$, the baby Skyrme profile $h$, and the gauge function $a$. We impose the following boundary conditions which guarantee a nontrivial topological charge:

$$
\begin{gathered}
h(r=0)=1, \quad h(R)=0, \quad h_{r}(R)=0, \\
a(r=0)=0, \quad a_{r}(R)=0, \\
\mathbf{B}(r=0)=1, \\
\mathbf{A}(r=0)=1,
\end{gathered}
$$

where the conditions for the derivatives of the Skyrme and gauge field come from the vanishing of the pressure at the compacton boundary. Here $R$ is a geometric size of the solitons, i.e., a value of the radial distance at which the matter field reaches its vacuum value. It can be finite (for the compactons) or infinite (for the usual infinitely extended solitons). Our choice of the condition on the metric function is motivated by an assumption that there is no conical singularity at the origin.

\section{B. The BPS property}

It is easy to notice that there is a formal solution corresponding to the zero pressure condition. Indeed,

$$
\mathbf{A}=1 \quad \text { and } \quad \tilde{p}=0
$$

solve two field equations (2.29) and (2.30). Now we have to solve the two remaining equations (2.28), (2.37) and show that the $\tilde{p}=0$ condition really leads to the solitonic solutions.

Before we go further, it is instructive to observe that in the zero pressure sector the Einstein-Hilbert action reduces to a pure boundary term, which obviously does not contribute to the equations of motion. Indeed, for $\tilde{p}=0$ (which enforces that $\mathbf{A}=1$ ) the curvature scalar simplifies to

$$
\mathbf{R}=-\frac{\mathbf{B}_{r}}{r \mathbf{B}^{2}} .
$$

Then, the Einstein-Hilbert action gives

$$
\begin{aligned}
S_{\mathrm{EH}} & =\frac{1}{\kappa^{2}} \int d^{3} x|g|^{1 / 2} \mathbf{R}=-\frac{1}{\kappa^{2}} \int d t d \varphi d r \frac{\mathbf{B}_{r}}{\mathbf{B}^{3 / 2}} \\
& =\frac{\pi T}{\kappa^{2}}\left(\mathbf{B}^{-1 / 2}(\infty)-\mathbf{B}^{-1 / 2}(0)\right),
\end{aligned}
$$

where $T$ is a time range for the temporal integral in the action. Now, let us perform a change of the radial variable and introduce

$$
\frac{d z}{d r}=r \sqrt{\mathbf{B}}
$$

The benefit of using the $z$ variable is that we can rewrite the full action of our model as

$$
S=-2 T M+\frac{\pi T}{\kappa^{2}}\left(\mathbf{B}^{-1 / 2}(\infty)-\mathbf{B}^{-1 / 2}(0)\right),
$$

where $M$ is the proper mass (energy) functional that is just the energy for the nongravitating gauged baby BPS Skyrme model. Gravity only contributes to the full action in the form of a boundary term. It is worthwhile to note that the above consideration is independent of a specific form of the matter field. The fact that, in the $\tilde{p}=0$ sector, the gravity can be reduced to a simple boundary term reflects the well-known fact that the $\mathrm{EH}$ action in $(2+1)$ dimensions is equivalent to a pure Chern-Simon theory. Of course, the full theory (with the matter field included) is not any longer reducible to a boundary part only.

After the above remark, we see that the introduction of the $z$ variable allows us to reduce the initial gravitating model to a nongravitating one, which is a genuine gauged BPS model with the following Bogomol'nyi equations:

$$
\begin{gathered}
n a_{z}=-e^{2} \lambda^{2} W(h), \\
\frac{n}{2}(1+a) h_{z}=-W_{h}(h),
\end{gathered}
$$

where $W=W(h)$ must obey a constraint following from the zero pressure equation, a so-called superpotential equation [17]

$$
\frac{e^{2} \lambda^{4}}{2} W^{2}+\lambda^{2} W_{h}^{2}=\mu^{2} \mathcal{U}(h)
$$

Since we consider potentials with a vacuum at $h=0$ (and possibly other isolated vacua), the superpotential equation enforces two boundary conditions at $h=0$, namely,

$$
W(h=0)=0, \quad W_{h}(h=0)=0 .
$$

The existence of a solution of this equation on the whole segment $h \in[0,1]$ obeying these boundary conditions is a 
rather nontrivial problem. Observe that in our construction the superpotential equation (2.49) does not show up from "nothing" as a necessary condition for the saturation of the Bogomol'nyi bound. Here, it is derived as the zero pressure condition that is at the very center of any BPS solution.

Earlier, we mentioned the proper mass of the matter, i.e., the energy of the soliton with the gauge field included [which is the nongravitational part of the total, i.e., asymptotic (ADM) mass]. One can define it as

$$
\begin{aligned}
M & =\int d^{2} x|g|^{\frac{1}{2}} \tilde{\rho} \\
& =\int d r d \varphi r \sqrt{\mathbf{B}}\left(\frac{n^{2}}{2 e^{2} r^{2} \mathbf{B}} a_{r}^{2}+\frac{\lambda^{2} n^{2}}{4 r^{2} \mathbf{B}}(1+a)^{2} h_{r}^{2}+\mu^{2} \mathcal{U}\right) \\
& =\int d z d \varphi\left(\frac{n^{2}}{2 e^{2}} a_{z}^{2}+\frac{\lambda^{2} n^{2}}{4}(1+a)^{2} h_{z}^{2}+\mu^{2} \mathcal{U}\right) \\
& =2 \pi|n| \lambda^{2}\left\langle W_{h}\right\rangle_{\mathbb{S}^{2}}=2 \pi|n| \lambda^{2}|W(h=1)|
\end{aligned}
$$

where $\left\langle W_{h}\right\rangle_{\mathbb{S}^{2}}$ is the $\mathbb{S}^{2}$ average of the superpotential. Here it is simply $\left\langle W_{h}\right\rangle_{\mathbb{S}^{2}}=\int_{0}^{1} W_{h} d h$. The second line shows that the proper mass is just the static energy functional of the gauged BPS baby Skyrme model in a flat space. The last equality comes from [17] and the fact that our solutions do obey the Bogomol'nyi equations and, therefore, the pertinent topological inequality is saturated. As a consequence, the proper mass is a linear function of the modulus of the topological charge as expected for a BPS system. Furthermore, the coefficient is uniquely given by the value of the superpotential at the antivacuum, i.e., at $h=1$, whose knowledge does not require one to find a particular solution but can be obtained from the superpotential (that is a target space) equation. In other words, the proper mass of the gravitating BPS baby Skyrmion is given by a geometric quantity.

One can also observe that the superpotential equation depends only on a one dimensionless combination of the coupling constants. Indeed, if we define a new superpotential

$$
\omega=\frac{\lambda}{\mu} W
$$

then (2.49) can be rewritten as

$$
\omega_{h}^{2}+\beta^{2} \omega^{2}=\mathcal{U}
$$

where the new dimensionless parameter is

$$
\beta^{2}=\frac{e^{2} \lambda^{2}}{2}
$$

Thus,

$$
M=2 \pi|n| \lambda \mu\left\langle\omega_{h}\right\rangle_{\mathbb{S}^{2}}=2 \pi|n| \lambda \mu|\omega(h=1)| .
$$

In the limit of vanishing gauge coupling constant $\beta=0$ we get $\omega_{h}=\sqrt{\mathcal{U}}$, which leads to the expression for the BPS baby Skyrme model [13]. For arbitrary $\beta$ the superpotential equation is a rather complicated nonlinear differential equation. However, as we show in the next section, it can be solved approximately with an arbitrary accuracy.

Next, we can use [17] and find the total magnetic flux carried by the baby Skyrmion. Namely,

$$
\begin{aligned}
\Phi & =\int d^{2} x|g|^{\frac{1}{2}} H=\int d r d \varphi r \sqrt{\mathbf{B}} \frac{n a_{r}}{r \sqrt{\mathbf{B}}} \\
& =2 \pi n \int d z a_{z}=2 \pi n a\left(z_{0}\right) \equiv 2 \pi n a_{\infty},
\end{aligned}
$$

where $z_{0}$ is the geometric size of the soliton (in the $z$ variable), which is finite for the compactons and infinite for the usual infinitely extended solitons. $H=\epsilon^{12} F_{12}=n a_{z}$ denotes the magnetic field. Hence, from the Bogomol'nyi equations one can find [17]

$$
a_{\infty}=-1+\exp \left(-\frac{F(1)}{4} \beta^{2}\right),
$$

where

$$
F(h)=4 \int_{0}^{h} \frac{W\left(h^{\prime}\right)}{W_{h^{\prime}}\left(h^{\prime}\right)} d h^{\prime}=4 \int_{0}^{h} \frac{\omega\left(h^{\prime}\right)}{\omega_{h^{\prime}}\left(h^{\prime}\right)} d h^{\prime}
$$

is a function of the target space variable, again uniquely defined for a given model (potential). Hence, again, the total flux can be found without solving the Bogomol'nyi equations but only by finding the superpotential.

Analogously, the geometric volume of the solitons reads

$$
\begin{aligned}
V= & \int d^{2} x|g|^{\frac{1}{2}}=2 \pi z_{0}=\pi \frac{\lambda}{\mu}|n| \exp \left(-\frac{F(1)}{4} \beta^{2}\right) \\
& \times \int_{0}^{1} \frac{\exp \left(\frac{F(h)}{4} \beta^{2}\right)}{\omega_{h}} d h .
\end{aligned}
$$

Of course, $z_{0}=V / 2 \pi$, which we will use later on.

The remaining piece is the equation for the metric function $\mathbf{B}$. It can be formally solved (in the $z$ radial variable)

$$
\mathbf{B}^{-1 / 2}(z)=1-\frac{\kappa^{2}}{2} \int_{0}^{z} \tilde{\rho}\left(z^{\prime}\right) d z^{\prime} .
$$

Obviously, as the metric function has to be a regular function we get a constraint

$$
1-\frac{\kappa^{2}}{4 \pi} \int_{0}^{z} 2 \pi \tilde{\rho}\left(z^{\prime}\right) d z^{\prime}>0 \Rightarrow \frac{\kappa^{2} M}{4 \pi}<1 .
$$

This condition is equivalent to the fact that the resulting metric has a deficit angle smaller than $2 \pi$ [23]. 
As a result, the magnetic gravitating BPS baby Skyrmions exist until a maximal topological charge $n_{\max }$,

$$
n_{\max }=\left\lfloor\frac{2}{\lambda \mu \kappa^{2}|\omega(1)|}\right\rfloor \text {. }
$$

This leads to a maximal proper mass, maximal magnetic flux, and maximal proper volume of our gravitating solitons. Specifically,

$$
M^{\max }=\frac{4 \pi}{\kappa^{2}}
$$

The proper mass (nongravitating mass), the magnetic flux, as well as the proper geometric volume are quantities that are linear in the topological charge. In fact, after the coordinate change they were obtained simply from the nongravitating gauged BPS baby Skyrme model. However, there are two "observables" that take into account the gravity interaction in a more nontrivial way. They are the total (asymptotic) mass and the radius. As we will see, both can also be obtained without the knowledge of the local form of the solutions, i.e., by proper target space integrals.

The ADM mass (total or asymptotic) in the $(2+1)$ dimensions is defined by the expression

$$
M_{\mathrm{ADM}}=\frac{4 \pi}{\kappa^{2}} \lim _{r \rightarrow \infty} m(r)
$$

where the radial function $m(r)$ in our ansatz for the metric is

$$
\frac{1}{B(r)}=1-2 m(r)
$$

It can be shown that the above definition leads to the integral formula

$$
\begin{aligned}
M_{\mathrm{ADM}} & =2 \pi \int_{0}^{R} r d r \tilde{\rho}(r)=2 \pi \int_{0}^{z_{0}} \frac{d z}{\sqrt{\mathbf{B}(z)}} \tilde{\rho}(z) \\
& =2 \pi \int_{0}^{z_{0}} d z \tilde{\rho}(z)\left(1-\frac{\kappa^{2}}{2} \int_{0}^{z} \tilde{\rho}\left(z^{\prime}\right) d z^{\prime}\right) .
\end{aligned}
$$

Hence,

$$
M_{\mathrm{ADM}}=2 \pi \int_{0}^{z_{0}} d z \tilde{\rho}(z)-2 \pi \frac{\kappa^{2}}{2} \int_{0}^{z_{0}} d z \tilde{\rho}(z)\left(\int_{0}^{z} \tilde{\rho}\left(z^{\prime}\right) d z^{\prime}\right) .
$$

However, the double integral can be written as

$$
\int_{0}^{z_{0}} d z \tilde{\rho}(z)\left(\int_{0}^{z} \tilde{\rho}\left(z^{\prime}\right) d z^{\prime}\right)=\frac{1}{2}\left(\int_{0}^{z_{0}} d z \tilde{\rho}(z)\right)^{2},
$$

which gives

$$
M_{\mathrm{ADM}}=M-\frac{\kappa^{2}}{8 \pi} M^{2}=M\left(1-\frac{\kappa^{2}}{8 \pi} M\right),
$$

where $M$ is the proper mass. Inserting (2.54) we find an exact formula

$$
M_{\mathrm{tot}}=2 \pi|n| \lambda \mu|\omega(h=1)|\left(1-\frac{\kappa^{2} \lambda \mu}{4}|n||\omega(h=1)|\right) .
$$

The total mass gets a correction due to the gravitational interaction that is quadratical with the topological charge. We remark that $M_{\mathrm{ADM}}$ grows with $n$ until $n=n^{\max }$ where $d M_{\mathrm{ADM}} / d n$ vanishes. In other words, the total mass instability occurs exactly at the maximal mass point. Hence, the maximal total mass is

$$
M_{\mathrm{ADM}}^{\max }=M_{\mathrm{ADM}}\left(n^{\max }\right)=\frac{M}{2}=\frac{2 \pi}{\kappa^{2}},
$$

which is one-half of the nongravitating mass.

It is interesting to notice that the relations between the $\mathrm{ADM}$ and proper mass (2.68), (2.70) are identical to the nongauged case. The unique place where the gauge interaction modifies our formulas is the value of the superpotential at the antivacuum $h=1$, which obviously changes if the gauge coupling constant changes.

Finally, the radius can be computed from

$$
\begin{aligned}
\frac{R^{2}}{2} & =\int_{0}^{R} r d r=\int_{0}^{z_{0}} \frac{d z}{\sqrt{\mathbf{B}(z)}} \\
& =\int_{0}^{z_{0}} d z\left(1-\frac{\kappa^{2}}{2} \int_{0}^{z} \tilde{\rho}\left(z^{\prime}\right) d z^{\prime}\right) .
\end{aligned}
$$

Thus,

$$
\frac{R^{2}}{2}=\frac{V}{2 \pi}-\frac{\kappa^{2}}{2} \int_{0}^{z_{0}} d z\left(\int_{0}^{z} \tilde{\rho}\left(z^{\prime}\right) d z^{\prime}\right) .
$$

In order to compute the double integral part, we have to turn to the topological bound. First of all, let us emphasize again that the static proper mass (energy) in the new radial coordinate $z$ is identical to the static energy functional of the gauged BPS baby Skyrme model in a flat space. Then, following the standard derivation of the Bogomol'nyi bound for the gauged BPS baby Skyrme model and using our axial static ansatz we find that (for simplicity we consider a positive topological charge and choose the sign of $W$ such that the integral is positive)

$$
\int_{0}^{z} \tilde{\rho}\left(z^{\prime}\right) d z^{\prime}=n \lambda^{2}(W(1)-W(h)-a(z) W(h)),
$$


where $h$ is understood as a function of $z$. If we integrate over the full domain of the solution, then $z=z_{0}$, which corresponds to $h=0$. But then $W(0)=0$, and we arrive at the usual total energy expression (divided by $2 \pi$ ). In the next step we use

$$
a(z)=-1+\exp \left(\frac{F(h(z))-F(1)}{4} \beta^{2}\right) .
$$

Moreover, any integral over the variable $z$ can be changed into a target space expression by

$$
d z=-\frac{n}{2} \frac{1+a}{W_{h}} d h=-\frac{n}{2} \frac{\exp \left(\frac{F(h)-F(1)}{4} \beta^{2}\right)}{W_{h}} d h .
$$

Finally, putting everything together we find

$$
\int_{0}^{z_{0}} d z\left(\int_{0}^{z} \tilde{\rho}\left(z^{\prime}\right) d z^{\prime}\right)=\frac{n^{2} \lambda^{2}}{2} \mathcal{A}(\beta),
$$

where

$$
\begin{aligned}
\mathcal{A}(\beta)= & \int_{0}^{1} \frac{\exp \left(\frac{F(h)-F(1)}{4} \beta^{2}\right)}{\omega_{h}} \\
& \times\left[\omega(1)-\exp \left(\frac{F(h)-F(1)}{4} \beta^{2}\right) \omega(h)\right] d h
\end{aligned}
$$

depends only on the superpotential, i.e., on a particular form of the potential. Note that the double integral, and therefore the gravity modification (shrinking) of the radius, is a quadratic function of the topological charge-exactly as in the nongauge case [19].

Now we can study the mass-radius relation. This can be performed by introducing the new variable $x=|n| / n_{\max } \in$ $[0,1]$. Then we find such a relation in a parametric way

$$
\left\{\begin{array}{l}
\frac{\kappa^{2} M_{\mathrm{ADM}}}{2 \pi}=x(2-x) \\
\frac{\kappa^{2} \mu^{2} R^{2}}{2}=\frac{\mathcal{A}(\beta)}{|\omega(1)|^{2}} x\left(\frac{\mathcal{C}(\beta)|\omega(1)|}{\mathcal{A}(\beta)}-x\right),
\end{array}\right.
$$

where

$$
\mathcal{C}(\beta)=\exp \left(-\frac{F(1)}{4} \beta^{2}\right) \int_{0}^{1} \frac{\exp \left(\frac{F(h)}{4} \beta^{2}\right)}{\omega_{h}} d h .
$$

Similar to [19] we define a new parameter $\Omega(\beta)$,

$$
\Omega(\beta)=\frac{\mathcal{C}(\beta)|\omega(1)|}{\mathcal{A}(\beta)} .
$$

Qualitatively, we obtain the same family of mass-radius curves as in the nongauge case $(\beta=0)$ [19] governed by the value of $\Omega$. For $\Omega=2, M_{\mathrm{ADM}}$ is a linear function of $R^{2}$. For $\Omega<2$ the $M_{\mathrm{ADM}}-R$ curve turns left at some value of the topological charge (or $x$ ) which means that the maximal radius does not coincide with the maximal mass. This is the case for $\Omega>2$, where the curve bends right.

Of course, one obvious question is whether the value of $\Omega$ can cross 2 while $\beta$ is changed. This would lead to a drastic change of the qualitative behavior of the mass-radius curve. We will investigate this issue taking the old baby potential.

It is a matter of fact that in the case of gravitating Skyrme models in $(3+1)$ dimensions there is a rather nontrivial bifurcation structure of solutions. Typically, there are two branches of solutions describing a self-gravitating Skyrmion: stable and unstable corresponding to smaller and bigger ADM mass, respectively. These two branches meet at a critical point (for example, a critical value of the gravitational coupling constant) beyond which no regular soliton exists [24]. Equivalently, one can find the two branches of solutions in the ADM mass-radius plot (with a fixed gravity coupling), where both ADM mass and radius are parametrized by the topological charge. This structure gets more complicated if more higher derivative terms are included-see [25] and especially [26]. However, we did not find such a pattern in the lower dimensional BPS baby Skyrme model. First of all, as we have shown before, in the zero pressure sector (relevant for all solutions presented here) the role of gravity can be reduced to a boundary term of the action, which does not contribute to the equations of motion. Then, we are left with the usual (nongravitating) gauged baby BPS model (in the redefined radial coordinate), for which no unstable solutions are known [17]. In principle, it could still be possible to have the gravitating solutions with a nonzero pressure (and the same topological charge as the $\tilde{p}=0$ solution) but they are ruled out by the same sequence of arguments as presented in [19]. Hence, only the zero pressure static solutions are allowed in the gravitating version of the gauged BPS baby Skyrme model. This guarantees that there are no unstable solutions.

\section{EXAMPLE-THE PIONIC MASS POTENTIAL}

\section{A. Superpotential}

As an example, we will consider the most popular old baby potential, which is a lower dimensional counterpart of the pionic mass potential for the Skyrme model

$$
\mathcal{U}_{\pi}=\frac{h}{4}
$$

We have to begin our analysis with the superpotential equation (2.52) whose knowledge is essential for the computation of all quantities

$$
\omega_{h}^{2}+\beta^{2} \omega^{2}=\frac{h}{4}, \quad \omega(0)=0 .
$$



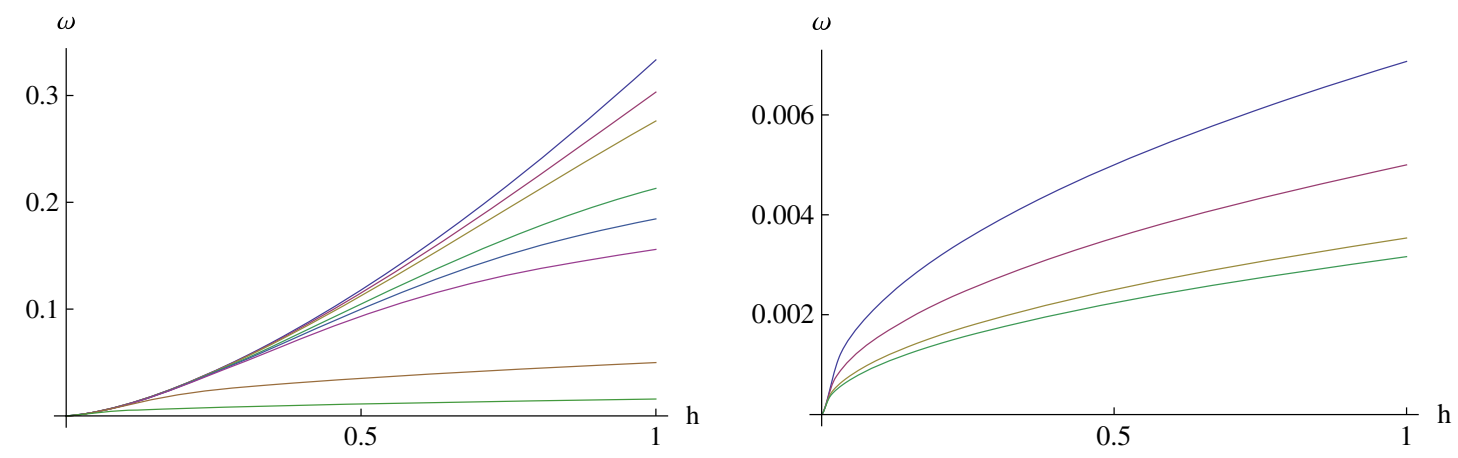

FIG. 1. Superpotential $\omega$ for the old baby potential (3.1). Left panel: $\beta^{2}=0,1,2,5,7,10,10^{2}, 10^{3}$. Right panel: $\beta^{2}=5 \times 10^{3}, 10^{4}$, $2 \times 10^{4}, 2.5 \times 10^{4}$. Increasing $\beta$ corresponds to a more suppressed curve.

Some numerical solutions to this equation in the unit segment $[0,1]$ are presented in Fig. 1. There are two limiting cases for which we can solve the equation exactly.

First, for $\beta=0$ we arrive at the nongauge case and

$$
\omega_{\beta=0}=\frac{1}{3} h^{3 / 2} .
$$

For a finite but small $\beta$ we may apply the perturbative expansion and find

$$
\begin{aligned}
\omega_{\text {small }}= & h^{3 / 2}\left(\frac{1}{3}-\frac{2}{63}(\beta h)^{2}+\frac{10}{6237}(\beta h)^{4}\right. \\
& \left.-\frac{92}{5893965}(\beta h)^{6}+o\left(\beta^{6} h^{6}\right)\right),
\end{aligned}
$$

which agrees extremely well with the numerics for $\beta^{2}<8$ on the whole unit segment. For higher values of the parameter the approximated solution begins to disagree in the vicinity of $h=1$. Once we increase $\beta$, such a disagreement is more and more pronounced and occurs for smaller $h$. This forces us to analyze an expansion at the $h=1$ end.

Observe first that for very large values of the parameter, $\beta \rightarrow \infty$, the superpotential equation gives $\omega=\frac{1}{2 \beta} h^{1 / 2}$. This provides an approximation close to $h=1$. For a finite but large $\beta$ we find the following approximated solution:

$$
\begin{aligned}
\omega_{\text {large }}= & h^{3 / 2}\left(\frac{1}{2}(\beta h)^{-1}-\frac{1}{16}(\beta h)^{-3}-\frac{13}{256}(\beta h)^{-5}\right. \\
& \left.-\frac{213}{2048}(\beta h)^{-7}+o\left(\beta^{-7} h^{-7}\right)\right) .
\end{aligned}
$$

Of course, it cannot serve as an approximated solution on the full segment, as its derivative is divergent at the origin. However, in the vicinity of $h=0$ the solution can always be approximate by the small $\beta$ solution. Because of that, the large $\beta$ approximated solution is

$$
\omega_{\text {approx }}=\left\{\begin{array}{ll}
\omega_{\text {small }} & h \in\left[0, h_{0}\right] \\
\omega_{\text {large }} & h \in\left[h_{0}, 1\right]
\end{array},\right.
$$

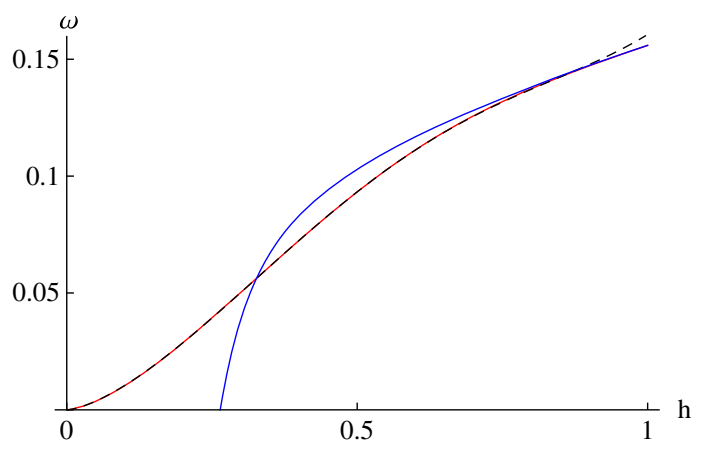

FIG. 2. The superpotential $\omega$ for the pionic potential (3.1) and $\beta^{2}=10$ (red line) with $\omega_{\text {small }}$ (dotted line) and $\omega_{\text {large }}$ (blue line) approximate functions.

where the gluing point $h_{0}$ is defined as

$$
\omega_{\text {small }}\left(h_{0}\right)=\omega_{\text {large }}\left(h_{0}\right),
$$

which, for the order of the expansion assumed above, is

$$
h_{0}=2.7821 \frac{1}{\beta}
$$

For too small $\beta$ the gluing point $h_{0}$ is not in the unit segment and, as a consequence, the approximated solution is given simply by the small $\beta$ expansion. This solution reproduces the true numerical solution with a great accuracy for all $\beta$. In Fig. 2 we show the numerical superpotential $\omega$ for $\beta^{2}=10$ (red curve) together with $\omega_{\text {small }}$ (dotted curve) and $\omega_{\text {large }}$ (blue curve). The correct approximation $\omega_{\text {large }}$ is provided by a composition of the dotted and blue curves glued at the second crossing point $h_{0}\left(\beta^{2}=10\right)=0.8798$.

\section{B. Masses, magnetic flux, proper geometric volume, and radius}

Using the value of the approximated superpotential at $h=1$ we can find the following approximate but analytical formula for the proper mass: 


$$
M=2 \pi|n| \lambda \mu \cdot \begin{cases}\frac{1}{3}-\frac{2}{63} \beta^{2}+\frac{10}{6237} \beta^{4}-\frac{92}{5893965} \beta^{6}+o\left(\beta^{6}\right) & \beta \leq 2.7821 \\ \frac{1}{2} \beta^{-1}-\frac{1}{16} \beta^{-3}-\frac{13}{256} \beta^{-5}-\frac{213}{2048} \beta^{-7}+o\left(\beta^{-7}\right) & \beta \geq 2.7821\end{cases}
$$

In Fig. 3 we plot an approximated $\omega_{\text {approx }}(1)$ as a function of the dimensionless parameter $\beta$. The gluing point is $h_{0}=1$, which corresponds to $\beta=2.7821$. The true numerical value is undistinguishable from the approximated (dashed) curve. Therefore, the approximated formula for the proper mass agrees with the true numerical curve with a very good accuracy. This formula is also sufficient to get an approximated but analytical expression for the ADM total mass. We just need (2.69).

In the next step we find an analytical, approximated expression for the magnetic flux. This requires knowledge of the function $F(h)(2.57)$

$$
\begin{aligned}
\frac{1}{4} F(h)= & \left(\frac{1}{3} h^{2}+\frac{4}{189} \beta^{2} h^{4}+\frac{32}{18711} \beta^{4} h^{6}+\frac{32}{280665} \beta^{6} h^{8}\right) \\
& \times \Theta\left[h_{0}-h\right]+\left(-1.76336 \beta^{-2}+\frac{61}{64} \beta^{-6} h^{-4}\right. \\
& \left.+\frac{5}{8} \beta^{-4} h^{-2}+h^{2}-\beta^{-2} \ln h-1.0232 \beta^{-2} \ln \beta\right) \\
& \times \Theta\left[h-h_{0}\right] .
\end{aligned}
$$

Hence, the flux is

$$
\frac{\Phi}{2 \pi n}=-1+\exp \left(-\beta^{2} \frac{F(1)}{4}\right)
$$

where

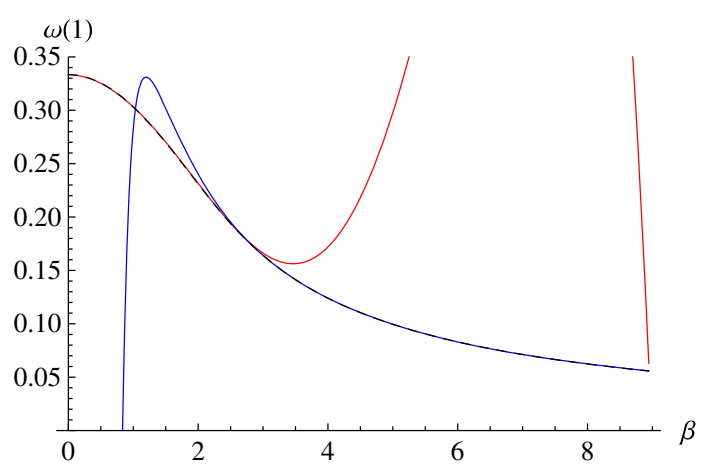

FIG. 3. The value of the superpotential at $h=1$, i.e., $\omega(1)=$ $M /(2 \pi|n| \lambda \mu)$ for the pionic potential (3.1) as a function of $\beta$. Dashed line: approximated expression; red line: $\omega_{\text {small }}(1)$; and blue line: $\omega_{\text {large }}(1)$.

$$
\begin{aligned}
\frac{1}{4} F(1)= & \left(\frac{1}{3}+\frac{4}{189} \beta^{2}+\frac{32}{18711} \beta^{4}+\frac{32}{280665} \beta^{6}\right) \\
& \times \Theta[2.7821-\beta]+\left(1-1.7634 \beta^{-2}\right. \\
& \left.+\frac{5}{8} \beta^{-4}+\frac{61}{64} \beta^{-6}-1.0232 \beta^{-2} \ln \beta\right) \\
& \times \Theta[\beta-2.7821] .
\end{aligned}
$$

It is worth noticing that for $\beta=2.7821$ (i.e., when $\omega_{\text {large }}$ must be taken into account) the flux is $\Phi /(2 \pi n)=$ -0.9936 , which is very close to its asymptotic value -1 . The approximated expression for the magnetic flux is plotted in Fig. 4.

Although the magnetic flux is practically quantized for $\beta>2.7821$, the proper geometric volume of compactons is still not too small. Specifically, it drops approximately 5 times from the nongauge case. An approximated formula for $\beta<2.7821$ is

$V=4 \frac{\lambda}{\mu}|n| \pi\left(1-\frac{2}{9} \beta^{2}+\frac{10}{567} \beta^{4}-\frac{92}{392931} \beta^{6}\right)$.

We plot it in Fig. 5.

We conclude that our approximation agrees very well with the numerical results. Of course, taking more terms in the small and large $\beta$ expansion of the superpotential we can approach an arbitrary accuracy, solving the model completely.

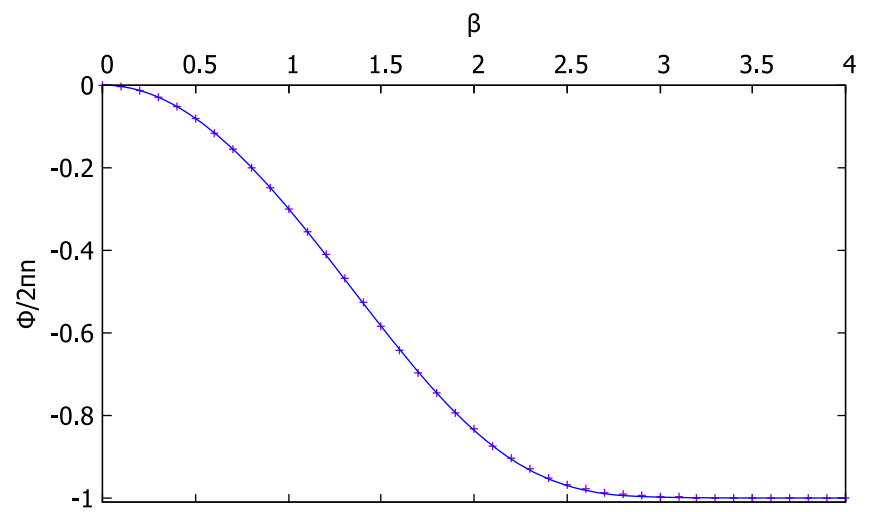

FIG. 4. The magnetic flux (left, blue line: approximated flux; violet points: numerical flux) for the old baby potential (3.1) as a function of $\beta$. 


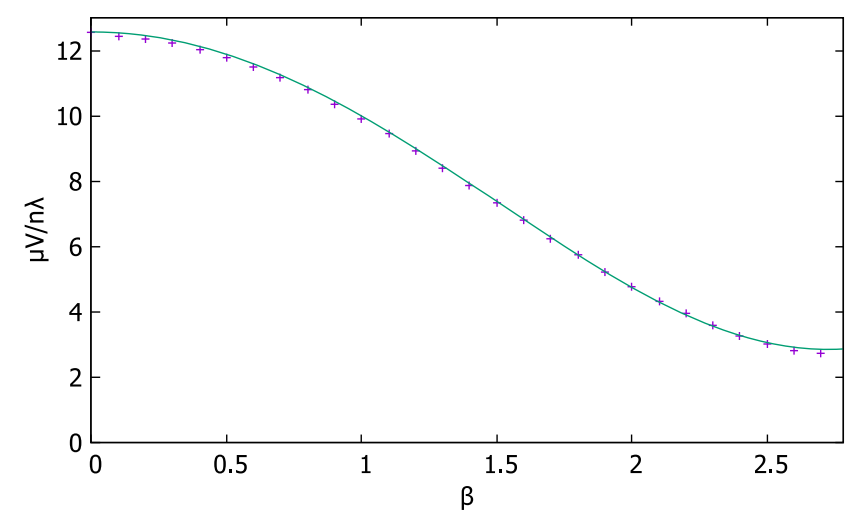

FIG. 5. The volume for the old baby potential (3.1) as a function of $\beta$ (violet points: numerical volume; green line: approximated formula).

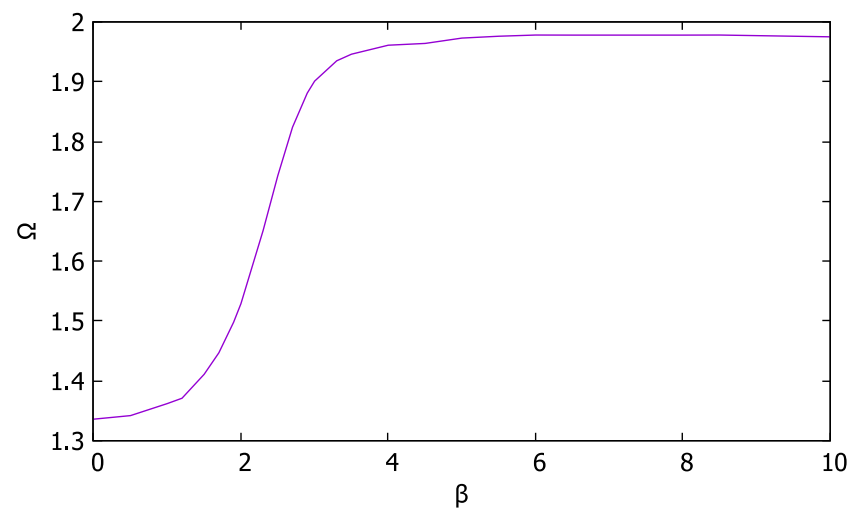

FIG. 6. $\Omega$ as a function of the coupling constant $\beta$ for the old baby potential (3.1).

\section{Mass-radius curve}

As we know, the shape of the mass-radius curve strongly depends on the value of $\Omega$. For the nongauged model it reads $\Omega(\beta=0)=4 / 3$. This means that the mass-radius curve bends at some point (the maximal radius point) toward the left. In fact, it has recently been observed that in the BPS baby Skyrme model $\Omega<2$ is a rather preferred

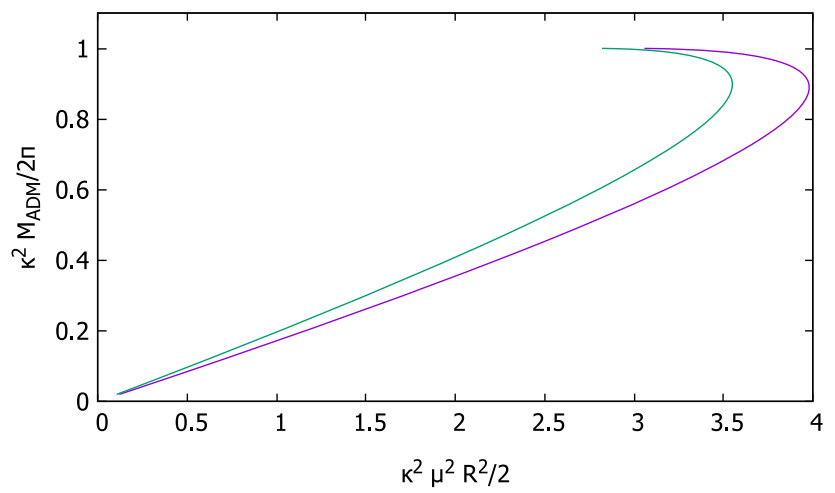

value for many one-vacuum potentials [19]. It is a matter of fact that for the old baby potential (3.1) $\Omega(\beta)$ is a growing function of the coupling $\beta$-see Fig. 6. It goes from $4 / 3$ and asymptotically reaches $\Omega=2$. This can be proven using the approximated superpotential. In fact, if $\beta \rightarrow \infty$, it is enough to take $\omega=\omega_{\text {large }}=\sqrt{h} /(2 \beta)$. Then, all possible corrections (from $\omega_{\text {small }}$ ) contribute to the $h \rightarrow 0$ end, which, because of the regularity of the integral, does not have any importance for the value of $\Omega(\beta=\infty)$.

A physical explanation of this asymptotical behavior of $\Omega$ is quite obvious. As the coupling constant grows, solitons become more and more squeezed, which physically means that the matter is more and more stiff with the energy density given by almost a step function. However, it is known that for the maximally stiff matter, i.e., the BPS baby Skyrme model with the Heaviside step potential, $\Omega=2$ and we arrive at the linear dependence between mass and radius squared. In Fig. 7 we plot the mass-radius curve for $\beta=0$ (nongauge case with $\Omega=4 / 3$ ), $\beta=1$ $(\Omega=1.36)$, and $\beta=4(\Omega=1.96)$.

Let us notice that the magnetic interaction lowers the proper mass. A related observation is that increasing $e$ increases the value of the maximal topological charge carried by the gravitating soliton. It is because $\omega(1)$ gets smaller in (2.61).

\section{Skyrme profile $\boldsymbol{h}$ and magnetic field $\boldsymbol{H}$}

The value of the superpotential $\omega(h=1)$ in the $z$ variable is equivalent to $\omega(h(z=0))$. This, together with (2.38) and (2.39), gives us the complete set of the initial conditions that allow us to solve the Bogomol'nyi equations (2.47), (2.48), and (2.49) by implementing a RK4 method.

As a result, we obtain the numerically constructed functions $h(z)$ and $a(z)$, but the gauge field $a(z)$ is not a physical object, so it is more suitable to consider a magnetic field $H(z)=n a_{z}$. Furthermore, a knowledge of the solutions $h(z)$ and $a(z)$ leads to energy density $\tilde{\rho}(z)$ (2.31), which lets us compute the metric function $\mathbf{B}(z)$ (2.59). At this point we can also return to an original radial

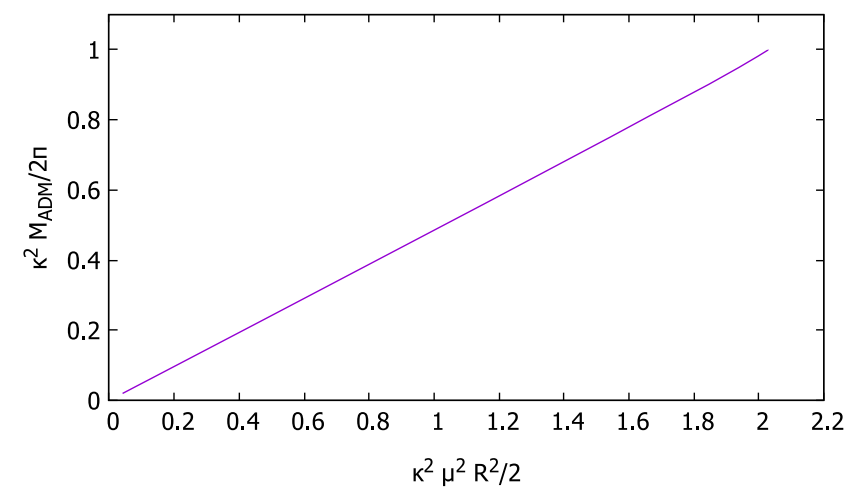

FIG. 7. The mass-radius square curve. Left: $\beta=0$ (violet line) and $\beta=1$ (green line). Right: $\beta=4$. 

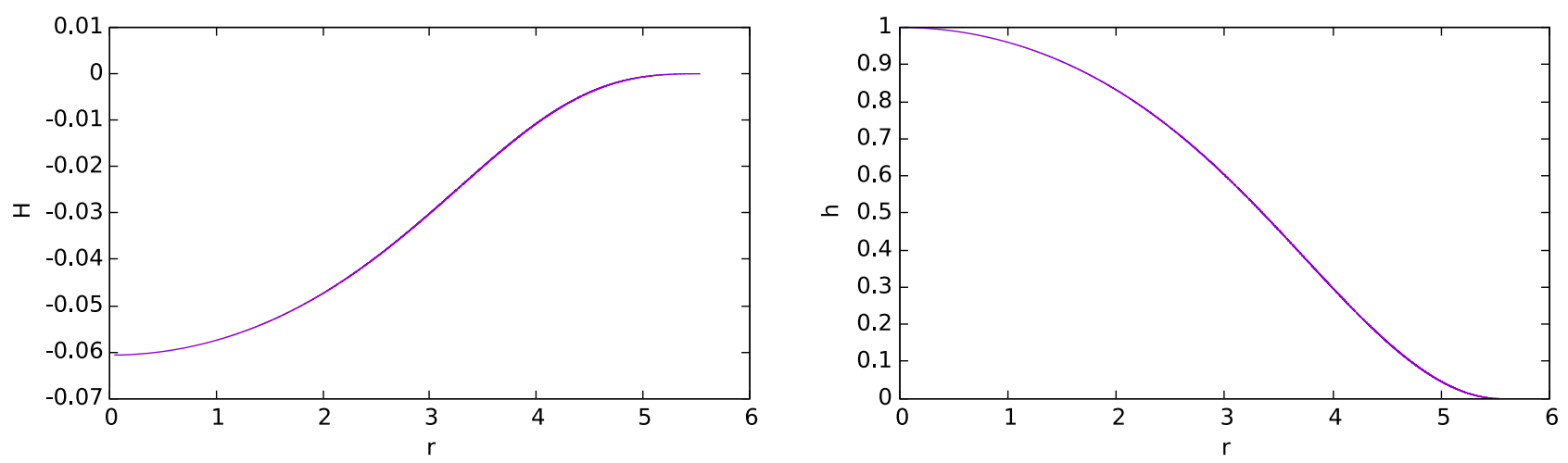

FIG. 8. Skyrme profile $h(r)$ and magnetic field $H(r)$ for the parameters $\lambda=1, \mu=0.1, e=\sqrt{2}$, and $\kappa^{2}=3$. Parameter $\beta^{2}=1$.
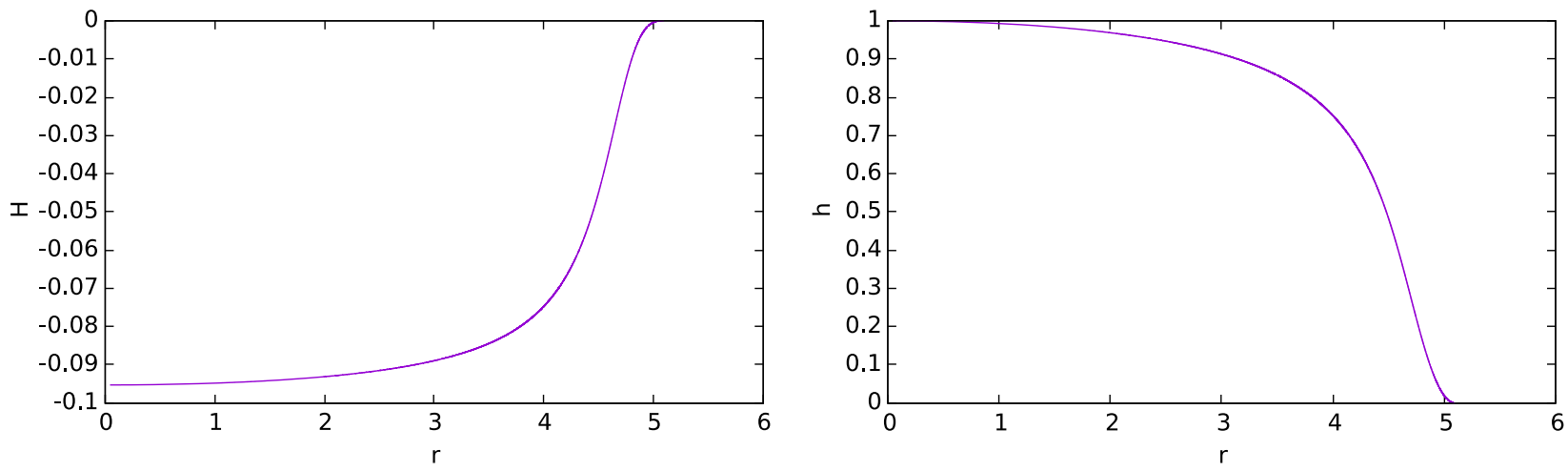

FIG. 9. Skyrme profile $h(r)$ and magnetic field $H(r)$ for the parameters $\lambda=\sqrt{5}, \mu=0.1, e=\sqrt{2}$, and $\kappa^{2}=3$. Parameter $\beta^{2}=5$.
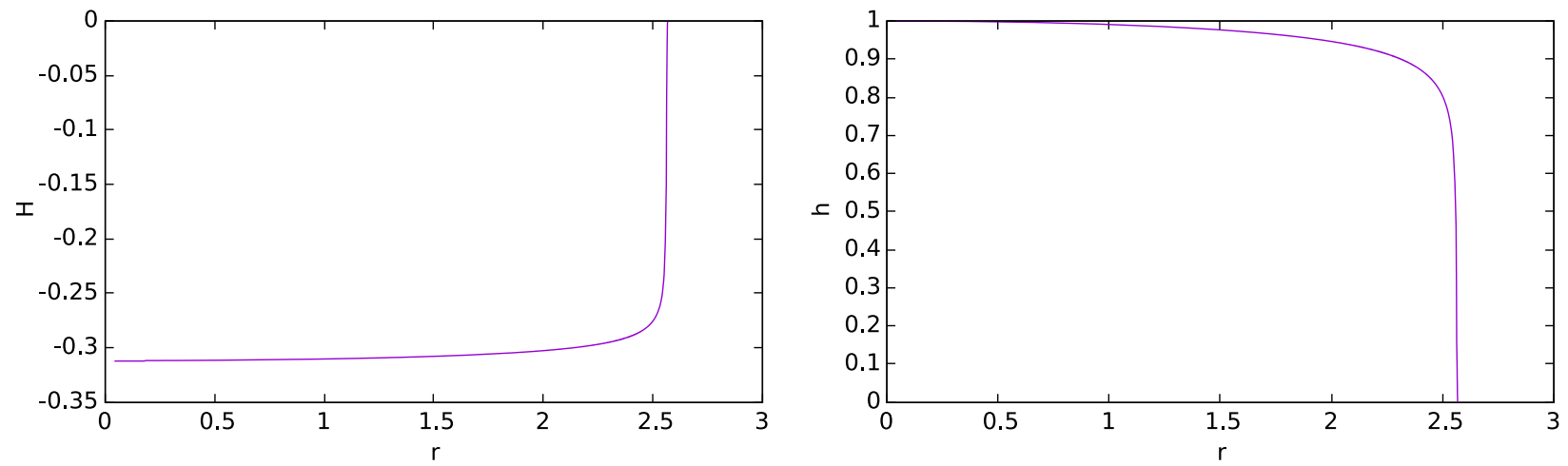

FIG. 10. Skyrme profile $h(r)$ and magnetic field $H(r)$ for the parameters $\lambda=1, \mu=0.1, e=\sqrt{20}$, and $\kappa^{2}=3$. Parameter $\beta^{2}=10$.

variable $r$ by performing a numerical integration. In Figs. $8-10$ we present the examples of the functions $h(r)$ and $H(r)$ [by functions $h(r)$ and $H(r)$ we mean the functions $h(z)$ and $H(z)$ after making an appropriate substitution] for a given set of the coupling constants.

As we see, the functions $H(r)$ and $h(r)$ are getting sharper as the parameter $\beta^{2}$ increases. This fact results in a bigger and bigger absolute value of both first derivatives $H_{r}$ and $h_{r}$ near the boundary $R$ of a soliton. If one wants to compute a superpotential $\omega(h)$ for a large $\beta^{2}$, then solving numerically the superpotential equation (3.2) brings no difficulty. However, the large values of a parameter $\beta^{2}$ force us to deal with fast changing functions near the boundary $R$ and thus making the numerical calculations of a profile $h(r)$ and a magnetic field $H(r)$ highly unstable. In conclusion, the fact that we can solely solve the superpotential equations (in the $h$ variable) and then based on that compute the observables grants us an opportunity to investigate much further the behavior of the observables as the functions of parameter $\beta$. 


\section{SUMMARY}

In this paper we investigated the BPS baby Skyrme model in $(2+1)$ dimensions coupled simultaneously to the Maxwell field and gravity. Such a theory, as we argued in the Introduction, might be used as a toy model of magnetized neutron stars.

The first main result is that such a self-gravitating theory of magnetized nonlinear matter with a nontrivial topology remains a BPS theory; that is, it supports solitonic solutions, being magnetized gravitating baby Skyrmions, as solutions of a zero pressure equation, and it admits a reduction to Bogomol'nyi equations where the proper (nongravitational) mass is a linear function of the topological charge. The corresponding topological lower bound on the proper mass integral is saturated. As a consequence, we derive a theory of magnetized and gravitating perfect fluid solitons in the $(2+1)$ dimension that is completely solvable, in the sense that all observables are given as some functions of the topological charge, with coefficients that are target space integrals depending on the coupling constant $\beta=e \lambda / \sqrt{2}$ and a particular model (particular potential). Hence, all observables are computable without any knowledge of the local form of solutions. This seems to be an expected result, as the underlying matter theory is a BPS theory in the nongravitating case. However, all observables obtained here that are nonlinear in the topological charge provide a new, analytical insight into the properties of gravitating magnetized solitons.

Specifically, the proper mass and proper geometric volume are the linear functions of the topological charge (assuming that the potentials lead to compact solitons). Next, the ADM mass as well as the radius squared get the negative gravitational corrections that are quadratic in the topological charge. This allowed us to completely classify the ADM mass-radius curves in the presence of magnetic flux. Interestingly, a nonzero value of the coupling constant $\beta$ (and therefore a nonzero value of the magnetic flux) modifies entirely the constants in the parametric massradius formula leaving the functional form unchanged. Again, as in the nongauge case $(\beta=0)$ the family of the curves can be divided into three rather distinguished groups, depending on whether the constant $\Omega(\beta)$ is smaller than, equal to, or bigger than 2 . Another feature that is not influenced by the inclusion of the magnetic field is the fact that the maximal ADM mass is one-half of the maximal proper mass.

Since the existence of the gravitating magnetized solitons is intimately related with the nongravitational case, we can conclude that there are no such solitons for the double vacuum potentials [for example, the so-called new baby potential $\mathcal{U}=\frac{1}{4} h(1-h)$ ]. Indeed, the gravitational interaction does not have any impact on the superpotential equation.

As all quantities rely on the knowledge of the superpotential, we developed a method of a derivation of it in an approximated but analytical way. We tested this expansion in the old baby potential case (3.1) and found perfect agreement. We believe that this approach can find some application for the study of the issue of the existence of the superpotential for an arbitrary field theoretical potential $\mathcal{U}$.

In addition, we constructed a numerical Skyrme profile $h(r)$ and a magnetic field $H(r)$ for the old baby potential (3.1) and the given set of the coupling parameters. The analysis of given solutions confirmed that solving the superpotential equation is not only an elegant way to investigate general properties of the observables but also it provides us with the high numerical stability.

From the physical point of view, our findings tell us that the modification of the mass-radius curve can be understood as flowing the baby Skyrmions toward more and more stiff matter.

There are many directions in which the current work can be continued. One can, for example, ask what happens if the Dirichlet (quadratic, i.e., $\sigma$-model) term is included. Especially it would be nice to understand how this influences the mass-radius curve. Of course, because of the lack of the axial symmetry (at least for the old baby potential [27]) this can be performed only within the meanfield approximation.

Obviously, the most important aim would be to investigate the self-gravitating magnetized BPS Skyrmions in the $3+1$ dimensions.

\section{ACKNOWLEDGMENTS}

The author thanks Andrzej Wereszczynski for discussion and Christoph Adam for comments.
[1] T. H. R. Skyrme, Proc. R. Soc. A 260, 127 (1961); Nucl. Phys. 31, 156 (1962).

[2] C. Adam, J. Sanchez-Guillen, and A. Wereszczynski, Phys. Lett. B 691, 105 (2010); Phys. Rev. D 82, 085015 (2010);
C. Adam, C. Naya, J. Sanchez-Guillen, and A. Wereszczynski, Phys. Rev. Lett. 111, 232501 (2013).

[3] E. Bonenfant and L. Marleau, Phys. Rev. D 82, 054023 (2010); E. Bonenfant, L. Harbour, and L. Marleau, Phys. 
Rev. D 85, 114045 (2012); M.-O. Beaudoin and L. Marleau, Nucl. Phys. B883, 328 (2014).

[4] C. Adam, C. Naya, J. Sanchez-Guillen, R. Vazquez, and A. Wereszczynski, Phys. Lett. B 742, 136 (2015); Phys. Rev. C 92, 025802 (2015).

[5] P. Bizon and T. Chmaj, Phys. Lett. B 297, 55 (1992); S. B. Gudnason, M. Nitta, and N. Sawado, J. High Energy Phys. 12 (2015) 013.

[6] C. Adam, M. Haberichter, and A. Wereszczynski, Phys. Rev. C 92, 055807 (2015).

[7] R. A. Battye, N. S. Manton, P. M. Sutcliffe, and S. W. Wood, Phys. Rev. C 80, 034323 (2009); D. T. J. Feist, P. H. C. Lau, and N. S. Manton, Phys. Rev. D 87, 085034 (2013).

[8] S. Nelmes and B. M. A. G. Piette, Phys. Rev. D 84, 085017 (2011); 85, 123004 (2012).

[9] P. Sutcliffe, J. High Energy Phys. 08 (2010) 019; 04 (2011) 45; C. Naya and P. Sutcliffe, J. High Energy Phys. 05 (2018) 174.

[10] M. Gillard, D. Harland, and M. Speight, Nucl. Phys. B895, 272 (2015); M. Gillard, D. Harland, E. Kirk, B. Maybee, and M. Speight, Nucl. Phys. B917, 286 (2017); S. B. Gudnason, Phys. Rev. D 93, 065048 (2016); S. B. Gudnason and M. Nitta, Phys. Rev. D 94, 065018 (2016); S. B. Gudnason, B. Zhang, and N. Ma, Phys. Rev. D 94, 125004 (2016).

[11] E. Witten, Nucl. Phys. B223, 422 (1983); 223, 433 (1983); C. G. Callan and E. Witten, Nucl. Phys. B239, 161 (1984).

[12] B. M. A. G. Piette, B. J. Schroers, and W. J. Zakrzewski, Z. Phys. C 65, 165 (1995); Nucl. Phys. B439, 205 (1995).

[13] C. Adam, T. Romanczukiewicz, J. Sanchez-Guillen, and A. Wereszczynski, Phys. Rev. D 81, 085007 (2010).
[14] J. M. Speight, J. Phys. A 43, 405201 (2010).

[15] T. Stępień, Geometric Methods in Physics, XXXI Workshop, Białowieża, Poland, 2012, Trends in Mathematics, edited by P. Kielanowski, S. Twareque-Ali, A. Odesskii, A. Odzijewicz, M. Schlichenmeier, and T. Voronov (Springer, Basel, 2013), pp. 229-237.

[16] T. Gisiger and M. B. Paranjape, Phys. Rev. D 55, 7731 (1997); A. N. Leznov, B. Piette, and W. J. Zakrzewski, J. Math. Phys. (N.Y.) 38, 3007 (1997); S. Bolognesi and W. Zakrzewski, Phys. Rev. D 91, 045034 (2015).

[17] C. Adam, C. Naya, J. Sanchez-Guillen, and A. Wereszczynski, Phys. Rev. D 86, 045010 (2012).

[18] T. Stępień, Acta Phys. Pol. B 46, 999 (2015).

[19] C. Adam, T. Romanczukiewicz, M. Wachla, and A. Wereszczynski, J. High Energy Phys. 07 (2018) 097.

[20] J. Gladikowski, B. M. A. G. Piette, and B. J. Schroers, Phys. Rev. D 53, 844 (1996); B. J. Schroers, Phys. Lett. B 356, 291 (1995).

[21] A. Samoilenka and Y. Shnir, Phys. Rev. D 93, 065018 (2016); 95, 045002 (2017); 97, 045004 (2018).

[22] F. Navarro-Lerida, E. Radu, and D. H. Tchrakian, Phys. Rev. D 95, 085016 (2017).

[23] P. Bizon and A. Wasserman, Phys. Rev. D 71, 108701 (2005).

[24] P. Bizon and T. Chmaj, Phys. Lett. B 297, 55 (1992).

[25] C. Adam, O. Kichakova, Y. Shnir, and A. Wereszczynski, Phys. Rev. D 94, 024060 (2016).

[26] S. B. Gudnason and M. Nitta, J. High Energy Phys. 05 (2018) 071.

[27] D. Foster, Nonlinearity 23, 465 (2010). 